\title{
Linear and Nonlinear Bullets of the Bogoliubov-de Gennes Excitations
}

\author{
S. Kumar, ${ }^{1}$ A. M. Perego, ${ }^{1,2, *}$ and K. Staliunas ${ }^{1,3}$ \\ ${ }^{1}$ Departament de Fisica, Universitat Politècnica de Catalunya, E-08222, Barcelona, Spain \\ ${ }^{2}$ Aston Institute of Photonic Technologies, Aston University, Birmingham B4 7ET, United Kingdom \\ ${ }^{3}$ Institució Catalana de Recerca i Estudis Avançats, Passeig Lluis Companys 23, E-08010, Barcelona, Spain
}

(Received 19 July 2016; published 27 January 2017)

\begin{abstract}
We report on the focalization of Bogoliubov-de Gennes excitations of the nonlinear Schrödinger equation in the defocusing regime (Gross-Pitaevskii equation for repulsive Bose-Einstein condensates) with a spatially modulated periodic potential. Exploiting the modification of the dispersion relation induced by the modulation, we demonstrate the existence of localized structures of the Bogoliubov-de Gennes excitations, in both the linear and nonlinear regimes (linear and nonlinear "bullets"). These traveling Bogoliubov-de Gennes bullets, localized both spatially and temporally in the comoving reference frame, are robust and propagate remaining stable, without spreading or filamentation. The phenomena reported in this Letter could be observed in atomic Bose-Einstein condensates in the presence of a spatially periodic potential induced by an optical lattice.
\end{abstract}

DOI: 10.1103/PhysRevLett.118.044103

The nonlinear Schrödinger equation (NLSE) is one of the most important equations of mathematical physics. It is a universal model for nonlinear dispersive waves in many conservative physical systems including fluids [1,2], optical fibers [3], plasmas [4], magnetic spin waves [5], and Bose-Einstein condensates. In the latter system, the NLSE is known as the Gross-Pitaevskii equation [6]. The NLSE, despite its apparent simplicity, supports a variety of fascinating nonlinear structures like solitons, breathers, recurrences, etc., hence being an attractive and rich model both in the description of natural phenomena and in technology-oriented research areas.

In particular, the localized solutions of the NLSE and its generalizations have attracted considerable attention, and a huge amount of the scientific literature has been devoted to characterizing their existence and stability in various spatial dimensions.

Written in a normalized form, the NLSE, for the temporal evolution of the field amplitude $A(\vec{r}, t)$ defined in space $\vec{r}$ and evolving in time $t$, reads

$$
\frac{\partial A}{\partial t}=i \nabla^{2} A+i c|A|^{2} A
$$

where $c$ is the nonlinearity coefficient and $\nabla^{2}$ the Laplace operator.

It is well known that the one-dimensional NLSE supports solitons in the strict mathematical sense, for focusing nonlinearity $(c>0)$. Zakharov and Shabat showed that a bright soliton solution exists [7]: In this case, the effects of nonlinear focusing and diffractive spreading are counterbalanced, and spatially localized nonlinear modes are stable. Bright solitons of the NLSE have been observed in nonlinear optics [8]. In fiber optics, solitons provide a powerful tool for optical communication systems [9].
If a finite field background is present, coherent structures localized in space and in time, exhibiting periodic oscillations, the so-called breathers, have been predicted analytically [10-12] and observed experimentally [13]. Furthermore, new solutions on the finite background can be constructed by using refined mathematical techniques [14] showing the incredible richness of the NLSE.

For $c<0$, the NLSE is defocusing, and its homogeneous solution (the condensate) is stable. In this case, the NLSE supports the existence of vortices and dark or gray solitons [8] but not the bright soliton. Simply speaking, this is because both the diffraction and the nonlinearity tend to spread (defocus) the excitations over the homogeneous background. There is no counterbalancing effect.

A modified NLSE, which includes dissipative and higherorder nonlinear terms, can support stable localized solutions [15]. Even though an analytical soliton solution cannot be found in these cases, such localized solutions are nevertheless called solitons in a broad sense of the definition.

The excitations of small amplitude waves on the homogeneous solution, $A_{0}$, are the so-called Bogoliubov-de Gennes (BdG) modes. They are generally more widely considered in the context of Bose-Einstein condensates (BECs) [16,17], where they correspond to density waves over the bulk homogeneous background.

The homogeneous field amplitude, perturbed by the weak Bogoliubov modes, can be written in the following form:

$$
\begin{aligned}
A(\vec{r}, t)= & A_{0} \exp \left(i c\left|A_{0}\right|^{2} t\right)\left[1+b_{+}(\vec{r}, t) \exp (i \vec{k} \cdot \vec{r})\right. \\
& \left.+b_{-}(\vec{r}, t) \exp (-i \vec{k} \cdot \vec{r})\right],
\end{aligned}
$$

where $\left|b_{+}\right|,\left|b_{-}\right| \ll\left|A_{0}\right|$. Substitution in Eq. (1) leads to the evolution equation for the excitation mode $b_{+}$and its coupled $b_{-}^{*}$ : 


$$
\begin{gathered}
\frac{\partial b_{+}}{\partial t}=-i k^{2} b_{+}+i c\left|A_{0}\right|^{2}\left(b_{+}+b_{-}^{*}\right), \\
\frac{\partial b_{-}^{*}}{\partial t}=i k^{2} b_{-}^{*}-i c\left|A_{0}\right|^{2}\left(b_{+}+b_{-}^{*}\right) .
\end{gathered}
$$

From Eqs. (3a) and (3b), one can obtain the dispersion relation of the $\mathrm{BdG}$ modes:

$$
\omega(k)= \pm k \sqrt{\left(k^{2}+2\left|A_{0}\right|^{2} c\right)},
$$

where $k=|\vec{k}|=\sqrt{k_{\|}^{2}+k_{\perp}^{2}}$, while $k_{\|}$and $k_{\perp}$ are, respectively, the wave vectors in the parallel and orthogonal directions with respect to the (arbitrarily selected) propagation direction of the $\mathrm{BdG}$ waves.

The BdG mode wave packet in the linear regime (small $c$ and when BdG excitations amplitudes are small with respect to the background) spreads along and transverse to the propagation direction due to parabolic dispersion. The spreading is even stronger in the nonlinear regime (large $c$ and/or large BdG excitation amplitudes) due to the defocusing nonlinearity. It is well known that a spatial, periodic modulation of the potential of the NLSE can strongly modify the dispersion [Eq. (4)], introducing forbidden frequency bands and modifying the slope and curvature of the dispersion curves. It is exploited, for example, in photonic crystals for self-collimation [18-20] and focusing effects [21,22]. When the sign of the curvature reverses, it allows the existence of certain localized structures, the band-gap solitons, even for defocusing nonlinearity $[8,22-24]$.

In this Letter, we explore numerically the effects of a periodic potential on the BdG excitations of the NLSE. Specifically, we show the existence of spatiotemporally localized (in the comoving reference frame), stable in propagation over a finite field background, bullets of the $\mathrm{BdG}$ excitations in both the linear and nonlinear regimes. The existence of bullets in nonlinear dispersive systems is due to a fine balance between the nonlinearity, spatial diffraction, and temporal dispersion and was first proposed by Silberberg for self-focusing nonlinearity [25]. Some experimental works have demonstrated the propagation of such light bullets [26,27], which have been found to be stable for finite time intervals. In this work, the substantial difference from the cases mentioned above [25-27] is that we find localized structures in the case of a defocusing nonlinear system with a spatially periodic potential. The localized structures discussed in this Letter also differ substantially from the two-dimensional spatially localized, time-stationary gap solitons, demonstrated in the NLSE with a periodic potential, close to the band edges [28,29]. There are two main differences between the BdG bullets demonstrated here and the gap solitons. First, gap solitons bifurcate from the edge of the band gap, where the curvature of the dispersion line is nonzero. Therefore, the gap solitons exist only in the nonlinear regime (when the nonlinearity compensates dispersion). The BdG bullets, as shown here, bifurcate from the inflection point of the dispersion curve, where the second derivative of $\omega(k)$ with respect to $k$ is zero or close to zero. This means that, while the dispersion and diffraction are zero, the nonspreading wave packets exist also in the linear case (for low intensity of the excitations: linear BdG bullets). In the nonlinear case, the weak curvature of the dispersion curve is compensated by the nonlinearity. And second, the wellknown gap solitons, as described in the literature, are localized wave packets on a zero background; i.e., it is the condensate itself, localized in space and time. The BdG excitations, on the other hand, are defined as density excitations of the nonzero background.

The numerical visualization of dispersion.-We consider the NLSE describing the evolution of a complex field $A(x, y)$ defined in two spatial dimensions with a $2 \mathrm{D}$ spatially modulated periodic "egg-box" potential:

$\frac{\partial A}{\partial t}=i \nabla^{2} A+i c|A|^{2} A+4 i m\left[\cos \left(q_{x} x\right)+\cos \left(q_{y} y\right)\right] A$,

where $q_{x}$ and $q_{y}$ are the modulation wave numbers along the directions $x$ and $y$, respectively, $m$ is the amplitude of the modulation, and the nonlinearity coefficient $c<0$ (defocusing regime).

With $\vec{k}=\left(k_{x}, k_{y}\right)$ being the spatial wave numbers and $\omega(\vec{k})$ the temporal frequency, the dispersion curves of the modulated NLSE system can be numerically extracted from the simulation of the perturbed NLSE [30]. On a homogeneous nonzero background, we introduce a random $\delta$-correlated perturbation and allow the system to evolve for a certain time $T$, recording the field amplitude $A(\vec{r}, t)$ values in the entire space and time domain. Its spectrum $\tilde{A}(\vec{k}, \omega)$ is obtained through a simple Fourier transformation.

For every fixed value of $\omega$, the set of obtained wave numbers $\left(k_{x}, k_{y}\right)$ constitutes the so-called isofrequency curves. In the absence of modulation (and for small nonlinearity $c$ ), the dispersion of the defocusing NLSE has the shape of a paraboloid, as given by (4), and the isofrequency curves are concentric circles with an increasing radius for increasing values of $\omega$. In this isotropic regime, the isofrequency lines have negative curvature, meaning that different components of a wave packet will acquire an angular phase dependence and any BdG excitations will spread.

The introduction of the spatially modulated potential breaks the isotropy near the band edges, by forbidding propagation in certain directions for some frequency bands. More interestingly, the curvature of the isofrequency lines changes from negative to positive as one approaches the band edge from below. The transverse inflection point (at $\omega \sim \omega_{s c}$ ), that is, the point of zero curvature (flat curve) where the sign changes from negative to positive, can sustain spatial solitons, i.e., beams which propagate without spreading (self-collimation). The different scenarios are depicted in 
Fig. 1, which shows a numerically extracted dispersion curve. When positive curvature is obtained (for $\omega=\omega_{f}$ ), we have a regime of anomalous diffraction, and focusing occurs.

Analogously, the chromatic dispersion curve [see Fig. 1(b)] also changes the sign of curvature, passing through an inflection point (flat curvature). A wave packet whose spectrum is tightly centered around the frequency $\omega_{\text {inf }}$ (longitudinal inflection point) will undergo little to no temporal spreading upon propagation and is called a temporal soliton. When the spatial and temporal soliton frequencies coincide, one can expect to have a spatiotemporal soliton, known in the literature as a "bullet" or a "light bullet" in the case of optics. Such bullets have previously been shown in a nonlinear, focusing medium, but neither in a defocusing medium nor on a finite nonzero field background.

Linear Bogoliubov-de Gennes bullets.-In the limit of weak nonlinearity, i.e., for small values of $c$ and/or $\left|A_{0}\right|^{2}$, the discussion presented in the previous section suggests that if one introduces a wave packet with the central wave vector corresponding simultaneously to both the transverse and longitudinal inflection points, on top of the finite background, then the wave packet will propagate without diffracting in space and without spreading in time. We call these solutions of the modulated NLSE linear BdG bullets.

In order to demonstrate the existence of the linear BdG bullets, we numerically introduce a wave packet of BdG excitations on the steady-state solution of the modulated NLSE [Eq. (5)] and observe its propagation. The first step therefore is to find the steady state, which corresponds to a spatially periodic Bloch mode with an (arbitrary) average amplitude $A_{0}$ and periodicities $q_{x}$ and $q_{y}$ in the $x$ and $y$ directions, respectively. An artificial transient step is needed to "cool" the system into the stationary Bloch solution.

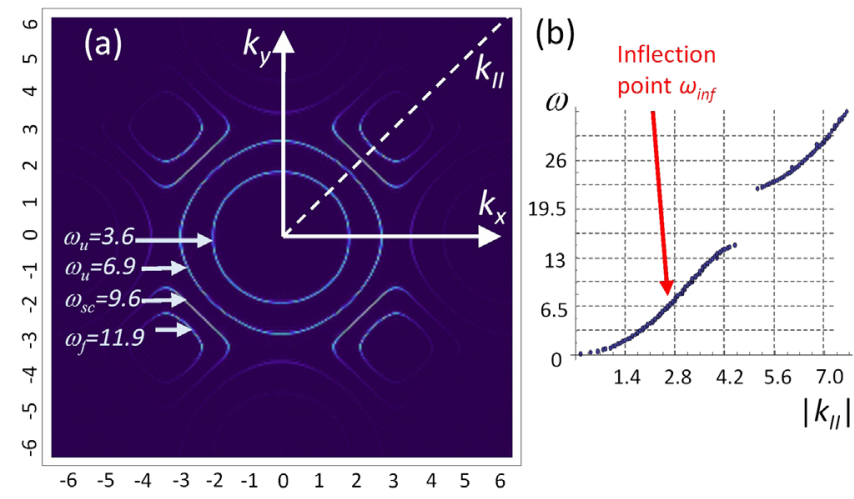

FIG. 1. (a) Numerically calculated 2D isofrequency curves for modulated NLSE in the linear regime for various different frequencies $\omega$ (where $\omega_{u}, \omega_{s c}$ and $\omega_{f}$ represent frequencies lying in the defousing, self-collimating and focusing regions respectively). The vertical cross section in the diagonal direction (along the dashed line) is plotted in (b), showing the band gap in that direction. The parameters used are $m=1, q_{x}=q_{y}=6.67$, $c=-0.05$, and $A_{0}=0.5$.
This is obtained by making a transformation to complex time, $t \rightarrow t(1+i \epsilon)$, with $0<\epsilon \ll 1$ and integrating Eq. (5)for a small period of time. This transformation mimics dissipation and causes damping of the thermal excitations of the condensate [31], allowing the system to relax to a stationary, spatially periodic Bloch mode, with average amplitude $A_{0}$.

Once the system has settled into the ground state, the complex time transformation is removed and the system switches back to the conservative regime. This time is referenced as $t=0$. Hereafter, we introduce the BdG bullet envelope over the steady-state background, which is a wave packet with a Gaussian envelope, having transverse and longitudinal widths given by $w_{\perp}$ and $w_{\|}$, respectively, amplitude $a_{0}$, and carrier wave vector $\vec{k}_{0}$ :

$$
a(\vec{r})=a_{0} \exp \left(\vec{k}_{0} \cdot \vec{r}\right) \exp \left[-\left(\frac{(x-y)^{2}}{w_{\|}^{2}}+\frac{(x+y)^{2}}{w_{\perp}^{2}}\right)\right] .
$$

The propagation dynamics of the BdG bullet is observed by integrating Eq. (5) in time. The diffractive and dispersive spreading of the pulse depends on the choice of the carrier wave vector $\vec{k}_{0}$. We can have a bullet solution, for an appropriate choice of $\vec{k}_{0}$, corresponding to both transverse and longitudinal inflection points, where the wave packet remains propagating without spatiotemporal spreading for arbitrarily long time durations.

However, Eq. (6) is not an exact bullet solution, which leads to substantial scattering when the bullet is introduced on the steady-state background. Because of periodic boundary conditions used in all the numerical simulations performed, the initial scattered radiation leads to strong noise in the system affecting the stability of the steady state and propagating pulse. For this reason, the scattered radiation is sufficiently, although not completely, filtered out of the system using a filtering mask around the central pulse, before allowing the bullet to propagate.

The choice of the wave vector $\vec{k}_{0}$ also imposes the direction of the pulse propagation. By contrast, in the unmodulated defocusing NLSE, any choice of $\vec{k}_{0}$ would result in a spreading of the wave packet. In Fig. 2, we demonstrate the stable propagation of the linear BdG bullet, in which the amplitude of the bullet has been chosen to be small compared to that of the background (see Fig. 2 parameters).

The propagation over a long time interval results in negligible dispersive or diffractive broadening of the bullet, while, on the other hand, a "normal" pulse (away from the inflection point) spreads over the entire background in this same time interval [Figs. 2(b) and 2(c)].

A movie showing the propagation of a linear bullet and a normal pulse is provided (see Supplemental Material, Movie 1 [32]).

Nonlinear self-focusing of wave packets.-If the nonlinearity coefficient $c$, the field intensity $|A|^{2}$, and/or the bullet amplitude $a_{0}$ are large, the nonlinearity has a more 
(a)
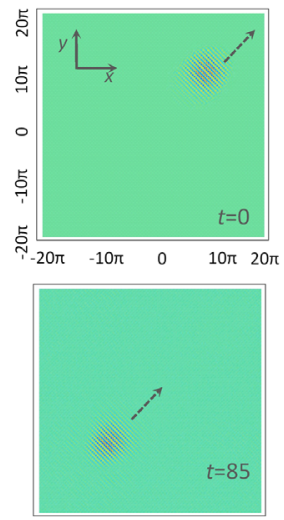

(b)
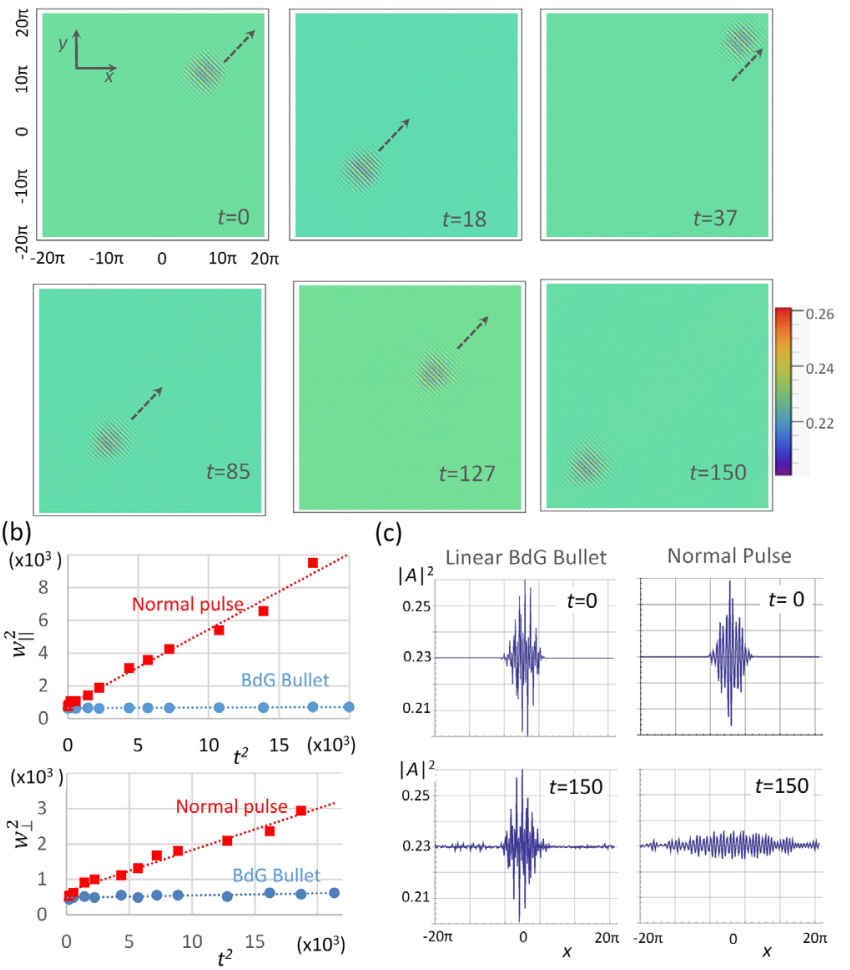

FIG. 2. (a) Snapshots of a linear BdG bullet propagation (for $c=-0.05$ and $a_{0}=0.05$ ), at different time intervals with $\vec{k}_{\text {bullet }}=2.45(\hat{x}+\hat{y})$, corresponding to the inflection point in Fig. 1 . The field intensity $|A|^{2}$ (without spatial modulations at wave numbers $q_{x}$ and $q_{y}$ ) is plotted. (b) Comparison of the widths of the BdG bullet, with a normal, diffracting pulse $\left[\vec{k}_{N P}=\right.$ $1.45(\hat{x}+\hat{y})]$. The normal pulse follows Gaussian diffraction (the dotted line follows: $w^{2} \propto t^{2}$ ), whereas the bullet propagates virtually unchanged. (c) Snapshots of the cross sections at $t=0$ and after a long time $(t=150)$ clearly show that the bullet propagates without spreading. Other parameters are $m=1, \quad q_{x}=q_{y}=6.67$, $A_{0}=0.5$, and initial widths $w_{\perp}=w_{\|}=18.5$.

pronounced effect. In the presence of an anomalous diffraction, the diffraction and nonlinearity play opposite roles, and generally three effects can occur-spreading, focusing, or confined propagation of a pulse-depending on the parameters. In Fig. 3, we show an example of selffocusing (filamentation) of large amplitude BdG excitations upon propagation.

Nonlinear Bogoliubov-de Gennes bullets. - The observation of the nonlinear focusing (Fig. 3), in both the longitudinal and transverse directions, is the prelude for the observation of the BdG bullets, which are spatiotemporally localized excitations formed by a superposition of nonlinear dispersive waves propagating on top of the finite nonzero background. At variance with the linear BdG bullets, the nonlinear bullets have an amplitude comparable to that of the background and are obtained for a much larger nonlinearity coefficient (see Fig. 4 parameters). We find indeed that, even in the presence of a considerable amount
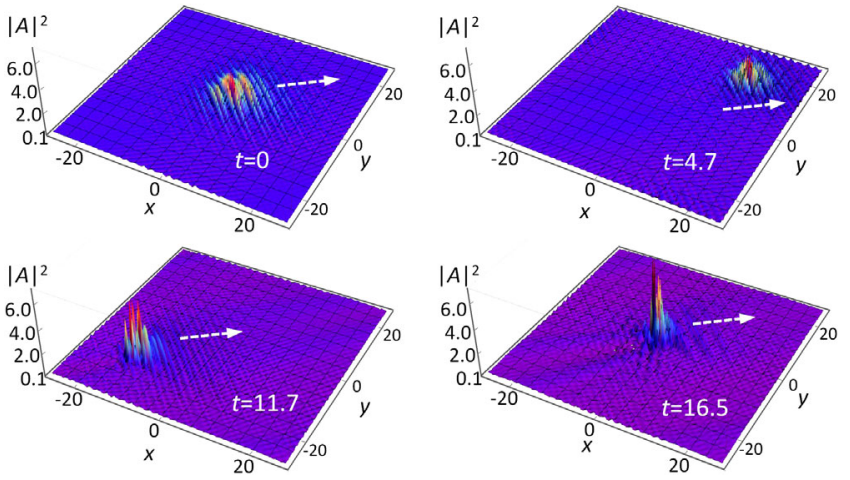

FIG. 3. Nonlinear self-focusing of a BdG wave packet. A highintensity Gaussian pulse is excited, with wave vector $\vec{k}_{0}=2.7(\hat{x}+\hat{y})$, lying close to the band edge in the anomalous diffraction regime. The field intensity $|A|^{2}$ (without spatial modulations at wave numbers $q_{x}$ and $q_{y}$ ) is plotted. The pulse strongly focuses upon propagation before ultimately breaking up (not shown here). Other parameters: $m=1, q_{x}=q_{y}=6.67, c=-0.1$, $A_{0}=0.5, a_{0}=1.1$, and initial widths $w_{\perp}=10$ and $w_{\|}=14$.

of nonlinearity, coherent structures localized in both spatial dimensions and in time, the nonlinear BdG bullets, can propagate without deformation for an arbitrarily long time due to the fine balancing between the nonlinearity and diffraction. The results are summarized in Fig. 4.

For solitons, there generally exists a family of solutions, relating the soliton width and amplitude. We explore numerically such families of soliton solutions, for different coefficients of nonlinearity $c$, by scanning the parameter space $\left(a_{0}, w\right)$, where $a_{0}$ is the amplitude and $w$ is the width of the beam.

We measure the scaling of the intensity versus the width of the soliton bullet, which is plotted in Fig. 4(a). The scaling obeys the following proportionality relation $I \propto 1 / w^{2}$, which agrees with the analytical predictions for solitons in general. This result, when plotted as a loglog graph, results in a straight line, as presented in Fig. 4(b).

One such solution (denoted by the black arrow) from the families of solutions is plotted in Fig. 4(c). The plots show snapshots of the bullet in the $x-y$ plane at different times. The excited wave packet at $t=0$ corresponds to the wave packet after filtering out the initial scattered radiation. We see that the shape and width of the pulse remain virtually unchanged through the temporal evolution. A movie showing the propagation of this nonlinear BdG bullet, in comparison to a diffracting pulse, is provided (Supplemental Material, Movie 2 [32]).

The stability of both linear and nonlinear BdG bullets is guaranteed by the fact that they coexist in their evolution, without breakup or deformation, with the residual (after filtering) scattered noise present in the system upon introduction of the bullet on the background. A quantitative analysis of the stability of the BdG bullets with respect to perturbative noise is provided in Supplemental Material [33]. 


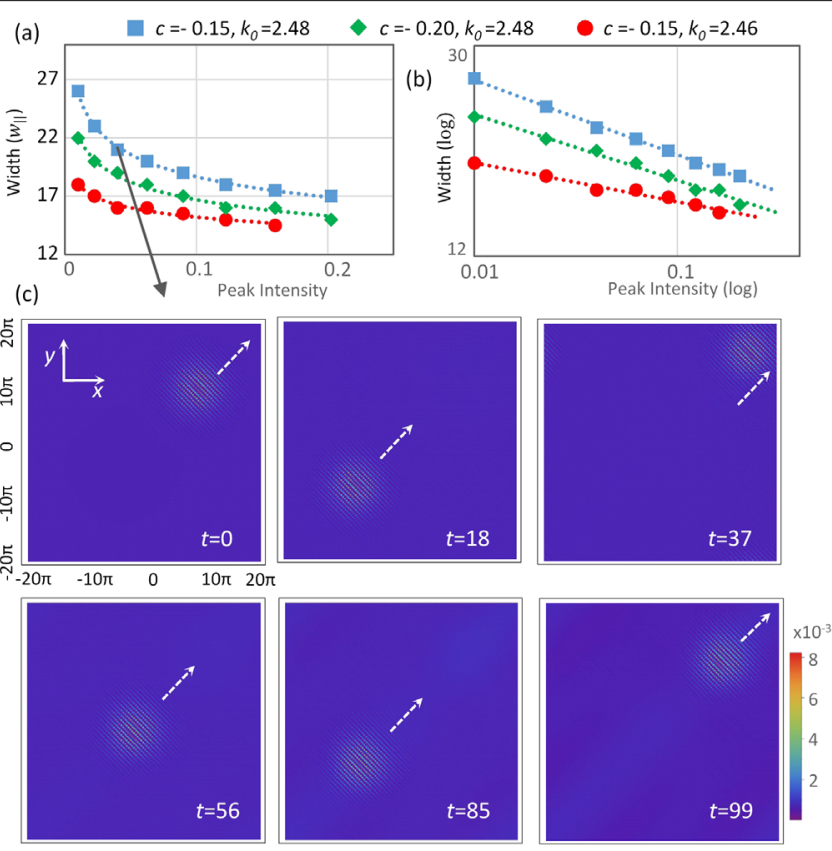

FIG. 4. (a) Family of solitons, for different parameter sets, showing the dependence of the pulse width versus the peak intensity. (b) The same plots in a log-log scale are a straight line, which fits well with the $I \propto 1 / w^{2}$ scaling relation for solitons. (c) Snapshots for a propagating nonlinear BdG bullet, where the field intensity $|A|^{2}$ (without spatial modulations at wave numbers $q_{x}$ and $q_{y}$ ) is plotted, at different time intervals. The bullet corresponds to one of the parameter sets in (a) as indicated by the black arrow. The dashed arrows show the direction of propagation. The parameters for the bullet are $a_{0}=0.2, c=-0.15, w_{\perp}=w_{\|}=21, m=1$, $q_{x}=q_{y}=6.67, A_{0}=0.5$, and $\vec{k}_{\text {NLbullet }}=2.48(\hat{x}+\hat{y})$.

Conclusions.-Through numerical simulations of the two-dimensional NLSE, in the defocusing regime with a spatially periodic potential, we have provided evidence for the self-focusing dynamics and localized bullets of the BdG excitations. The most critical condition for obtaining the bullets is the choice of the central wave vector, which should correspond to both transverse and longitudinal inflection points.

The BdG bullets could be possibly observed experimentally in defocusing atomic BECs in the presence of a periodic potential induced by an optical lattice.

The work is financially supported by Spanish Ministerio de Educación y Ciencia, European FEDER (Fondo Europeo de Desarrollo Regional) through Project No. FIS2015-65998-C2-01 and by the NATO SPS985048 grant. A. M. P. acknowledges support of the FP7 ITN Marie-Curie Action, Project ICONE (608099).

*Corresponding author. peregoa@aston.ac.uk

[1] M. J. Ablowitz, Nonlinear Dispersive Waves (Cambridge University Press, Cambridge, England, 2011).
[2] A. Osborne, Nonlinear Ocean Waves and the Inverse Scattering Transform (Academic, Boston, 2010).

[3] G. P. Agrawal, Nonlinear Fiber Optics (Academic, San Diego, 2006).

[4] V. E. Zakharov, Zh. Eksp. Teor. Fiz. 62, 1745 (1972) [Sov. Phys. JETP 35, 908 (1972)].

[5] A. K. Zvedzin and A. F. Popkov, Sov. Phys. JETP 57, 350 (1983).

[6] L. P. Pitaevskii and S. Stringari, Bose-Einstein Condensation (Clarendon, Oxford, 2003).

[7] V. E. Zakharov and A. B. Shabat, Zh. Eksp. Teor. Fiz. 1, 118 (1971) [Sov. Phys. JETP 34, 62 (1972)].

[8] Y.S. Kivshar and G. P. Agrawal, Optical Solitons (Academic, San Diego, 2003).

[9] L. P. Mollenauer and J. P. Gordon, Solitons in Optical Fibers, Fundamentals and Applications (Elsevier Academic, Amsterdam, 2006).

[10] N. N. Akhmediev, V. M. Eleonskii, and N. E. Kulagin, Zh. Eksp. Teor. Fiz. 89, 1542 (1985) [Sov. Phys. JETP 62, 894 (1985)].

[11] E. A. Kuznetsov, Dokl. Akad. Nauk SSSR 236, 575 (1977) [Sov. Phys. Dokl. 22, 507 (1977)].

[12] D. H. Peregrine, J. Aust. Math. Soc. Series B, Appl. Math. 25, 16 (1983).

[13] B. Kibler, J. Fatome, C. Finot, G. Millot, F. Dias, G. Genty, N. Akhmediev, and J. M. Dudley, Nat. Phys. 6, 790 (2010).

[14] V. E. Zakharov and A. A. Gelash, Phys. Rev. Lett. 111, 054101 (2013).

[15] Dissipative Solitons, edited by N. Akhmediev and A. Ankiewicz (Springer, Berlin, 2005).

[16] Y. Castin and R. Dum, Phys. Rev. A 57, 3008 (1998).

[17] Y. Castin, in Lecture Notes of Les Houches Summer School, edited by R. Kaiser, C. Westbrook, and F. David (EDP Sciences and Springer-Verlag, Berlin, 2001), pp. 1-136.

[18] H. Kosaka, T. Kawashima, A. Tomita, M. Notomi, T. Tamamura, T. Sato, and S. Kawakami, Appl. Phys. Lett. 74, 1212 (1999).

[19] D. N. Chigrin, S. Enoch, C. M. Sotomayor Torres, and G. Tayeb, Opt. Express 11, 1203 (2003).

[20] K. Staliunas and R. Herrero, Phys. Rev. E 73, 016601 (2006).

[21] J. Trull, L. Maigyte, V. Mizeikis, M. Malinauskas, S. Juodkazis, C. Cojocaru, M. Rutkauskas, M. Peckus, V. Sirutkaitis, and K. Staliunas, Phys. Rev. A 84, 033812 (2011).

[22] Nonlinear Photonic Crystals, edited by R. E. Slusher and B. J. Eggleton (Springer, Berlin, 2003).

[23] J. W. Fleischer, M. Segev, N. K. Efremidis, and D. N. Christodoulides, Nature (London) 422, 147 (2003).

[24] O. Morsch and M. Oberthaler, Rev. Mod. Phys. 78, 179 (2006).

[25] Y. Silberberg, Opt. Lett. 15, 1282 (1990).

[26] S. Minardi et al., Phys. Rev. Lett. 105, 263901 (2010).

[27] X. Liu, L. Qian, and F. W. Wise, Phys. Rev. Lett. 82, 4631 (1999).

[28] E. A. Ostrovskaya and Y. S. Kivshar, Phys. Rev. Lett. 90, 160407 (2003).

[29] B. Eiermann, Th. Anker, M. Albiez, M. Taglieber, P. Treutlein, K.-P. Marzlin, and M. K. Oberthaler, Phys. Rev. Lett. 92, 230401 (2004). 
[30] E. Gaizauskas, A. Savickas, and K. Staliunas, Opt. Commun. 285, 2166 (2012).

[31] M. L. Chiofalo, S. Succi, and M. P. Tosi, Phys. Rev. E 62, 7438 (2000).

[32] See Supplemental Movies 1 and 2 at http://link.aps.org/ supplemental/10.1103/PhysRevLett.118.044103. In both movies, the field intensity $|A|^{2}$ (without spatial modulations at wave numbers $q_{x}$ and $q_{y}$ ) and its cross section along the $x$ direction are plotted. Parameters of Movie 1: $c=-0.05$, $a_{0}=0.05$, and $w_{\perp}=w_{\|}=18.5$. The central wave vector for the spreading normal pulse is $\vec{k}_{N P}=1.45(\hat{x}+\hat{y})$, while for the stable linear bullet it is $\vec{k}_{\text {bullet }}=2.45(\hat{x}+\hat{y})$.
Parameters of Movie 2: $c=-0.15, a_{0}=0.2$, and $w_{\perp}=w_{\|}=19$. The central wave vector for the spreading pulse is $\vec{k}_{N P}=1.45(\hat{x}+\hat{y})$, while for the stable nonlinear bullet it is $\vec{k}_{\mathrm{NLbullet}}=2.48(\hat{x}+\hat{y})$. The common parameters used in both movies are $A_{0}=0.5, m=1$, and $q_{x}=q_{y}=6.67$; the $2 \mathrm{D}$ integration area is $40 \pi \times 40 \pi$, with a $256 \times 256$ discretization grid, and periodic boundary conditions are used.

[33] See Supplemental Material at http://link.aps.org/ supplemental/10.1103/PhysRevLett.118.044103 for a quantitative study demonstrating the stability of the BdG bullets with respect to noise. 